\title{
CLINICAL RESEARCH ARTICLE An experience with a bubble CPAP bundle: is chronic lung disease preventable?
}

\author{
Hany Aly ${ }^{1,2}$ and Mohamed A. Mohamed ${ }^{1}$
}

BACKGROUND: Continuous positive airway pressure (CPAP) is associated with marginal decrease in chronic lung disease (CLD). This study aims to report outcomes, with focus on CLD, of preterm infants managed with a bubble CPAP (b-CPAP) bundle of care. METHODS: Infants $<1500 \mathrm{~g}$ were stratified into four groups depending on intubation status through first 3 days of life. The incidence of mortality, CLD and other morbidities were compared over four chronological epochs. Outcomes of the most recent epoch were compared to contemporaneous benchmarks from Vermont Oxford Network (VON).

RESULTS: Of 773 infants (median $G A=28$ weeks, average $B W=995 \mathrm{~g}$ ), $24.5 \%$ were intubated in DR and $11.7 \%$ in the first day of life. Mechanical ventilation, bCPAP and oxygen days in survivors were 1.5, 29 and 14, respectively. Overall incidence of CLD was $6.4 \%$ that remained consistent in the four epochs $(7.6 \%, 7.5 \%, 5.8 \%$ and $5 \%)$, respectively. In comparison to VON, CLD was significantly less $(p<0.001)$. Initial DR intubation was not associated with increased CLD compared to initial management with CPAP that required subsequent intubation.

CONCLUSION: It is feasible and sustainable to administer a b-CPAP bundle of care to decrease CLD. Cluster randomized trials are needed to validate the reproducibility of this approach.

Pediatric Research (2020) 88:444-450; https://doi.org/10.1038/s41390-020-0763-3

\section{INTRODUCTION}

Chronic lung disease (CLD) continues to lead neonatal morbidities in premature infants despite surfactant discovery and the emergence of newer modalities for mechanical ventilation. ${ }^{1}$ The use of mechanical ventilation and oxygen supplementation are the two major attributes to CLD. ${ }^{2}$

The use of continuous positive airway pressure (CPAP) as the primary mode of respiratory support in premature infants has recently increased. In addition to avoiding ventilator-induced lung injury, CPAP optimizes the functional residual capacity of the lungs, splints the airways and diaphragm, and stimulates lung growth; these effects have the potential to minimize the risk for CLD. ${ }^{3}$ Multiple randomized controlled trials compared early use of CPAP to mechanical ventilation and did not show difference in the primary outcome of CLD. ${ }^{4,5}$ Consequently, the American Academy of Pediatrics considered it an acceptable practice to support premature infants with CPAP in the delivery room (DR) without routine intubation and surfactant administration. ${ }^{6}$ However, when these trials were analyzed collectively in a meta-analysis, the early use of CPAP demonstrated a marginal but statistically significant decline in the composite outcome of survival without CLD. ${ }^{7}$

As much as these trials were crucial to clarify the safety of not routinely intubating premature infants in $D R$, and therefore they were instrumental for the change in DR management and practice, these trials left multiple questions unanswered. Significant variations in the incidence of CLD were reported among these trials despite recruiting infants with comparable inclusion criteria and using the same definition of CLD. In addition, the majority of these trials were not able to reproduce the success previously reported in anecdotal experiences. For example, in neonatal units experienced with the use of bubble CPAP (b-CPAP), very-low-birth-weight (VLBW) infants were reported to have oxygen requirement at 36 weeks in $4-6 \%$ of infants with combined mortality and CLD of $13-17 \%{ }^{8,9}$ However, in randomized trials, the combined outcome of mortality and oxygen use at 36 weeks was mostly around $30 \%$ or higher. ${ }^{10}$ Therefore, CPAP as a tool is not able to explain the wide variation in CLD among neonatal units. In fact, in a recent report, significant increase in long-term pulmonary morbidities were associated with increased use of noninvasive ventilation during infants' stay in the neonatal intensive care unit (NICU). ${ }^{3}$

We hypothesize that it is not the CPAP tool that decreases CLD; what matters the most is the bundle of care associated with the use of CPAP. The bundle would include: (a) device-related guidelines: type of facial interface, the source of delivered CPAP pressure, the timing of starting and weaning off CPAP, the method of weaning CPAP, (b) bedside skills and competencies: positioning of the infant, maintenance of patent airway, keeping nasal interface in place and maintaining sealed respiratory circuits, and (c) nonpulmonary management: early fluid management, feeding practices, nutritional goals, and transfusion practices. We therefore described the components of this bundle of care in our experience and provided the outcomes associated with this overall bundle. This study was designed to achieve two goals: (1) distinguish between the CPAP bundle of care and the CPAP device, and (2) to set the precedence of associating a respiratory bundle of care, rather than a device, with clinical outcomes. We also compared our recent outcomes using this bubble CPAP bundle with contemporaneous outcomes from Vermont Oxford network to assess the effectiveness of this bundle of care. Consequently, future trials that will use CPAP would need to

\footnotetext{
${ }^{1}$ The George Washington University Hospital, Washington, DC, USA and ${ }^{2}$ Cleveland Clinic Children's Hospital, Cleveland, OH, USA
} Correspondence: Mohamed A. Mohamed (mmohamed@mfa.gwu.edu)

Received: 24 June 2019 Revised: 1 January 2020 Accepted: 3 January 2020

Published online: 17 January 2020 
specify the exact CPAP bundle and practice guidelines used to ensure uniformity among participating units.

\section{PATIENTS AND METHODS}

Patients

All VLBW infants $(B W<1500 \mathrm{~g})$ admitted since January 1, 2002 through December 31, 2017 were included in this study. The NICU was a level 3 unit that admitted inborn infants delivered in a highrisk university hospital setting. The population was divided into four epochs, 4 years each, in chronological order of admission. Analyses of outcomes over time were conducted to test the stability of these outcomes. The overall population was stratified into four groups depending on the status of respiratory support in the delivery room and during the first 3 days of life. These four respiratory groups were: Group 1 received CPAP in DR and continued with b-CPAP for at least 3 days; Group 2 received bCPAP in DR but were subsequently intubated within 3 days; Group 3 were initially intubated in DR and successfully extubated within 3 days; and Group 4 were intubated in DR and remained intubated for at least 3 days. The decision to use b-CPAP or to intubate VLBW infants was determined by the following b-CPAP bundle.

The bubble CPAP bundle of care

This bundle included the following items:

1. Delivery room management: Infants were not routinely intubated in the delivery room. Premature infants were allowed a chance to spontaneously breathe after tactile stimulation followed by $30 \mathrm{~s}$ of bag-and-mask ventilation if needed. If an infant had spontaneous breathing, he/she would be supported with CPAP using the t-piece connector (Carden valve or Neopuff, Fisher \& Paykel, Auckland, New Zealand) and would be immediately transported to the NICU and supported by b-CPAP while other care elements were established such as attaching cardiac monitoring, temperature check and anthropometric measurements. ${ }^{9}$

2. Early use of CPAP: The b-CPAP circuit was the primary type of CPAP used in the NICU. Detailed description of the circuit components was previously reported (Fig. S1). ${ }^{11}$ CPAP pressure was maintained at $5 \mathrm{mH}_{2} \mathrm{O}$ that could increase to $6 \mathrm{cmH}_{2} \mathrm{O}$ if an infant experienced retractions or increased oxygen requirement. The facial interface was always via nasal prongs; the prongs were snuggly fitted into both nostrils. Prongs were short to allow the least resistance and curved to follow the anatomy of the nares.

3. Use of surfactant: if an infant did not have spontaneous breathing in the delivery room, he/she would be intubated. Surfactant therapy would be administered only in the presence of oxygen requirement and radiographic findings suggestive of respiratory distress syndrome. Intubated infants were extubated as soon as acidemia and hypercapnia improved and oxygen requirement $\left(\mathrm{FiO}_{2}\right)$ was weaned to $<30 \%$.

4. CPAP failure criteria: While supported with b-CPAP, if an infant showed signs of respiratory compromise including frequent apnea or increased oxygen requirement $\left(\mathrm{FiO}_{2} \geq\right.$ $50 \%$ ), he or she would be intubated and started on conventional mechanical ventilation. Surfactant would be administered if tracheal intubation occurred in the first $24 \mathrm{~h}$ of life. An infant might benefit from a trial of noninvasive positive pressure ventilation (NIPPV) via nasal prongs prior to intubation if the compromise was mainly related to apnea.

5. Airway care: infants were closely monitored for patency of airway including the use of the right-sized nasal prongs and deep suctioning of the nasal and oral pharynx. Suction was applied gently every $3-4 \mathrm{~h}$ to avoid any harm to nasal mucosa. The position of the nasal prongs and respiratory circuit tubing were secured to a snug head cap to ensure the prongs do not impose any pressure or trauma on the nasal septum. An indwelling orogastric tube was used to prevent abdominal distension and evacuate gastric air during care every $3-4 \mathrm{~h}$. A neck roll was used to maintain the neck slightly extended. The use of b-CPAP did not prohibit positioning the infant prone or on the side as long as airways were maintained patent. A checklist that included detailed b-CPAP care competencies was maintained at the bedside for the caregiver to complete each shift. Staff were trained on the application of each component of the checklist at the bedside.

6. Weaning off CPAP: Infants were supported with b-CPAP until they no longer required oxygen supplementation and breathed comfortably. In addition, those with birth weight $<1000 \mathrm{~g}$ were maintained on b-CPAP, even if their $\mathrm{FiO}_{2}$ requirement was $21 \%$, until their weight was at least $1200 \mathrm{~g}$ and post-conceptual age was at least 32 weeks. Infants were weaned off b-CPAP directly to room air. If an infant displayed any sign of distress such as tachypnea, retraction, or oxygen desaturation, b-CPAP would be restarted and no weaning trials were attempted for $24 \mathrm{~h}$. Daily weaning attempts would continue until infant was fully weaned off CPAP. Infants were not weaned to nasal cannula or any other source of oxygen or airflow.

7. Use of caffeine: Infants $\leq 26$ weeks of GA were routinely started on caffeine, otherwise caffeine was administered to infants only if they were symptomatic with apnea or irregular breathing. Once started, infants were continued to receive caffeine until their post-conceptual age was close to 35 weeks and they were no longer supported with bCPAP. A few infants might be weaned earlier when they had tachycardia or gastritis.

8. Oxygen saturation: The lower monitor alarms were set at $85 \%$. High monitor alarms were set at $95 \%$ when $\mathrm{FiO}_{2}$ is $>21 \%$. Oxygen saturation range was attempted at $87-93 \%$ with narrower targeted range of $90-93 \%$.

9. Fluid and nutrition management: For infants $<1000 \mathrm{~g}$, fluids were started at volume of $120 \mathrm{ml} / \mathrm{kg} / \mathrm{d}$ and the goal might change every $8 \mathrm{~h}$ in response to point-of-care serum sodium concentrations. Aggressive parenteral nutrition approach was followed with protein intake of $1-2 \mathrm{~g} / \mathrm{kg} / \mathrm{d}$ in the first $24 \mathrm{~h}$ of life. Early trophic feeds were started and goal feeds of $130 \mathrm{Kcal} / \mathrm{kg} / \mathrm{d}$ was achieved at 14-21 days of life based on BW and clinical conditions. Fluid intake for infants with BW $>1000 \mathrm{~g}$ were initiated at $100 \mathrm{ml} / \mathrm{kg} /$ day with less frequent electrolyte checks.

10. Transfusion practices: Infants with $B W<1000 \mathrm{~g}$ were managed with liberal transfusion practice attempting to maintain hematocrit $\geq 40 \%$ during the first week of life and $\geq 35 \%$ after 1 week. Infants with $B W \geq 1000 \mathrm{~g}$ were maintained with hematocrit $\geq 35 \%$ during the first week of life and $\geq 30 \%$ thereafter.

Changes in practice. Over the 16 years of the study some changes to the practice were introduced. Symptomatic patent ductus arteriosus (PDA) was medically and then surgically treated that has changed in 2010 when no PDA treatment has been attempted since. ${ }^{12}$ Transfusion practice has changed in 2008 where feeding were withheld during red cell transfusion. ${ }^{13}$

Data collection

This was a retrospective analysis of prospectively collected data for infants with birth weight $(\mathrm{BW}<1500 \mathrm{~g})$. We excluded infants with chromosomal disorders and multiple complex congenital anomalies. Data items collected for this analysis included maternal 
age, hypertension (chronic and pregnancy induced), diabetes mellitus (all types), intra-amniotic infections (chorioamnionitis), prenatal steroids, multiple gestation, and mode of delivery, Apgar scores at 1 and 5 min, infants' sex, BW, GA, intubation in the delivery room and at day of life $0,1,2,3,7,14$ and any thereafter, surfactant and caffeine treatment, number of blood transfusions during hospital stay, postnatal steroids, bloodstream infections, pulmonary hemorrhage, intraventricular hemorrhage (IVH) (by ultrasound imaging), periventricular leukomalacia (PVL) (by ultrasound at 42 days of age or magnetic resonance imaging (MRI) at 36 weeks post-conceptual age or beyond), retinopathy of prematurity (ROP) (per retina specialist assessment), necrotizing enterocolitis (NEC) (medical and surgical), CLD defined as need for supplemental oxygen $\left(\mathrm{O}_{2}\right)$ at 36 weeks post-conceptual age, mode and duration of noninvasive and invasive ventilation (bubble CPAP, noninvasive positive pressure ventilation, conventional and high frequency mechanical ventilation), pneumothorax (by X-ray findings), chest tube placement and duration, length of hospital stay (LOS), and disposition at discharge (home, transfer for surgical indications, or death). Data collection and analysis were approved by the George Washington University Institutional Review Board.

\section{Analytic plan}

We calculated outcomes for the overall sample and for BW categories $1000-1499 \mathrm{~g}, 750-999 \mathrm{~g}, 500-749 \mathrm{~g}$, and $<500 \mathrm{~g}$. Maternal demographics and clinical findings, infants' demographic and clinical outcomes were calculated using frequency and normal distribution analyses. We used Mantel-Haenszel, Chisquare, and Fisher exact tests to calculate prevalence of mortality, out transport, CLD, and overall survival. We used $t$ tests to compare differences in means for continuous variables including days of intubation, b-CPAPVNIPPV, and oxygen therapy. Wilcoxon's rank sum test was used to compare nonparametric variables. Logistic regression analysis was used to compare outcomes of different respiratory interventions. All analyses were performed using SAS version 9.4 (Cary, NC). The primary outcome of this study was survival until hospital discharge without CLD. Secondary outcomes included duration of noninvasive and mechanical ventilation, LOS, prevalence of $N E C, I V H, P V L, R O P$, pulmonary hemorrhage and discharge weight, head circumference and length.

Specific aims

1. To assess the effectiveness of the described bubble CPAP bundle: the reported outcomes in this population were compared to a publically available contemporaneous benchmark outcomes from Vermont Oxford network (VON). Outcomes from VON data were used for the year 2014. ${ }^{14}$ This report included infants with BW 501-1500 g, therefore the outcomes of 196 infants (501-1500 g) in our sample born in the 2014-2017 period were compared to this benchmark VON sample. Respiratory and nonrespiratory outcomes were compared. The definition of CLD in this specific aim and in the VON benchmark report was similar, that was the use of oxygen at 36 weeks post-conceptual age or, if transferred or discharged at 34-35 weeks, were receiving supplemental oxygen at discharge. ${ }^{14}$

2. To assess sustainability of the bubble CPAP bundle: Respiratory management and outcomes were analyzed under four chronological periods, 4 years each, to test consistency of management and related outcomes. Data were expressed in mean (SD) and median (IQR) as appropriate. Statistical significance was detected when $p<0.05$.

3. To examine the relationship between early respiratory management and outcomes: We further compared outcomes according to early respiratory management in DR and through the first 3 days of life. Consequently, infants were categorized under four respiratory strategies: Group 1 received CPAP in DR and continued with b-CPAP for 3 days; Group 2 received $b$-CPAP in DR but were subsequently intubated within 3 days; Group 3 were initially intubated in DR and successfully extubated within 3 days; and Group 4 were intubated in DR and remained intubated for at least 3 days. Respiratory and nonrespiratory outcomes were compared. Regression analysis was used to control for demographic and clinical confounding variables.

\section{RESULTS}

A total of 773 infants with $B W<1500 \mathrm{~g}$ were included in this study. Maternal and neonatal demographics and clinical characteristics are presented in Table 1. The prevalence of CLD in the overall population was $6.4 \%$. Table 2 presents mortality, CLD and secondary outcomes by BW.

Outcomes for infants born in the years 2014-2017 were compared to a published sample of VON outcome data. ${ }^{14}$ CLD was significantly lower in GW compared to VON (4.8\% vs. $28.6 \%, p$ value $<0.001$ ) (Table 3 ). The total percentage of infants who were supported by b-CPAP with or without oxygen supply at 36 weeks were 10.3\%; comparison with benchmark could not be made as data were not available in VON report. Figure 1 presents mortality, CLD and survival rates in 4 years epochs from 2002 to 2017 showing sustainability of outcomes over the 16 years.

Table 4 demonstrates data on early respiratory management. Intubation in the DR was mainly driven by the degree of prematurity with $67 \%$ of infants' $\leq 24$ weeks were intubated in DR while $<20 \%$ in infants $>26$ weeks received DR intubation.

Survival without CLD differed according to respiratory support groups; the rates were $95.4 \%, 79.4 \%, 69.1 \%$ and $44.4 \%$ in groups 1 , 2,3 and 4 respectively (Table S1). After controlling for demographic and clinical confounding variables including maternal diabetes mellitus or hypertension, prenatal steroids, delivery route, multiple gestation, sex, and GA, the adjusted odds ratio (aOR) for survival without CLD did not differ between respiratory groups 2 and 3 (aOR $=0.40, \mathrm{Cl}: 0.16-1.02, p=0.055)$. However, it was different between groups 2 and 4 ( $\mathrm{aOR}=0.33, \mathrm{Cl}$ : $0.12-0.97$, $p=0.043)$. Survival without CLD was decreased in males $(\mathrm{aOR}=$ $0.50, \mathrm{Cl}: 0.27-0.93, p=0.029)$ and in SGA infants $(\mathrm{aOR}=0.20, \mathrm{Cl}$ : $0.09-0.47, p<0.001)$.

\section{DISCUSSION}

This study shows a consistent single-digit prevalence of CLD in VLBW infants managed with the bubble CPAP bundle. Infants who failed CPAP and became intubated within 3 days did not differ from those who were initially intubated in the DR followed by extubation in the first 3 days of life. The prevalence of ROP was much diminished with this management strategy without any ROP intervention over the last 8 years of the study period. Except for significantly less CLD at GW, reported outcomes were comparable between VON and GW for infants with BW 501-1500 g.

Bubble CPAP was the only type of CPAP used in this experience. There have been multiple theories to explain possible advantages for the use of b-CPAP compared to other types of CPAP. Bubbling at the expiratory end of the circuit generates a low-amplitude, high frequency pressure waves that varies according to the level and frequency of water oscillation; and could contribute to lung recruitment in premature infants. ${ }^{15}$ Although such explanation is biologically plausible, studies that examine lung recruitment with the use of b-CPAP compared to conventional CPAP are lacking. A single study showed improved oxygenation when using b-CPAP compared to ventilator-driven CPAP when randomly assigned to the same infant. ${ }^{16}$ Meanwhile, in the absence of studies to support 
Table 1. Maternal and infants demographics and clinical characteristics.

\begin{tabular}{|c|c|c|c|c|c|}
\hline & $\begin{array}{l}\text { All infants } \\
<1500 \mathrm{~g} \\
n=773\end{array}$ & $\begin{array}{l}\text { Infants } \\
1000-1499 \mathrm{~g} \\
n=397\end{array}$ & $\begin{array}{l}\text { Infants } \\
750-999 \mathrm{~g} \\
n=162\end{array}$ & $\begin{array}{l}\text { Infants } \\
500-749 \mathrm{~g} \\
n=165\end{array}$ & $\begin{array}{l}\text { Infants } \\
<500 \mathrm{~g} \\
n=49\end{array}$ \\
\hline Maternal age, year ${ }^{\mathrm{a}}$ & $31(26-35)$ & $31(25-35)$ & $31(27-35)$ & $30(25-35)$ & $30(26-35)$ \\
\hline Maternal diabetes mellitus & 9.7 & 11.3 & 8.6 & 5.4 & 14.3 \\
\hline Maternal Hypertension & 28.6 & 30.5 & 25.3 & 23.0 & 42.9 \\
\hline Prenatal steroids & 60.4 & 57.9 & 62.4 & 63.6 & 63.3 \\
\hline \multicolumn{6}{|l|}{ Infants demographics } \\
\hline Female infants & 52.26 & 49.6 & 51.9 & 55.2 & 65.3 \\
\hline Race/Ethnicity & \multicolumn{5}{|c|}{$\begin{array}{l}\text { Caucaseans } 16 \% \\
\text { African Americans } 69 \% \\
\text { Hispanics/Latinos 5\% } \\
\text { Asians 5\% } \\
\text { Others 5\% }\end{array}$} \\
\hline Singleton pregnancy & 74.4 & 73.1 & 73.3 & 76.4 & 75.5 \\
\hline Cesarean section delivery & 35.6 & 33.8 & 35.8 & 44.2 & 20.4 \\
\hline Apgar at $1 \mathrm{~min}^{\mathrm{a}}$ & $5(3-7)$ & $6(4-8)$ & $5(3-6)$ & $4(2-6)$ & $3(1-5)$ \\
\hline Apgar at $5 \mathrm{~min}^{\mathrm{a}}$ & $8(6-8)$ & $8(7-9)$ & $7(6-8)$ & $7(5-8)$ & $6(4-8)$ \\
\hline Gestational age, week $^{a}$ & $28(25.4-30)$ & $30(28.3-31)$ & $27(26-28)$ & $24.3(23.5-25.4)$ & $24(23.2-26)$ \\
\hline Birth weight, $g^{b}$ & $995 \pm 323$ & $1270 \pm 146$ & $867 \pm 76$ & $623 \pm 74$ & $443 \pm 51$ \\
\hline SGA/IUGR & 17.6 & 13.4 & 9.3 & 17.0 & 81.6 \\
\hline Respiratory distress syndrome & 90.0 & 84.9 & 95.1 & 100 & 100 \\
\hline Surfactant therapy & 21.4 & 8.1 & 23.6 & 42.4 & 51.0 \\
\hline Xanthine therapy & 66.4 & 45.8 & 80.3 & 94.9 & 97.7 \\
\hline
\end{tabular}

efficacy of other CPAP methods to reproduce superior outcomes, it is wise to continue using b-CPAP only and not to assume that all CPAP devices are equal.

The pressure used in the bubble CPAP bundle is $5 \mathrm{mH}_{2} \mathrm{O}$ that occasionally would increase to $6 \mathrm{cmH}_{2} \mathrm{O}$ if an infant exhibited increased oxygen requirements, severe chest retraction and increased work of breathing. The practice did not allow for a pressure higher than $6 \mathrm{cmH}_{2} \mathrm{O}$. Some studies associated the use of higher pressure $\left(8 \mathrm{cmH}_{2} \mathrm{O}\right)$ with a significant increase in the risk to develop pneumothorax. ${ }^{5}$ The greater incidence of pneumothorax with the use of $8 \mathrm{cmH}_{2} \mathrm{O}$ pressure could be a marker that such pressure is higher than physiologic and would be concerning to impose trauma to alveoli even in the absence of pneumothorax. The pressure used in the DR was also $5-6 \mathrm{cmH}_{2} \mathrm{O}$ without routine bag-and-mask ventilation or sustained positive end expiratory pressure (PEEP) in order to avoid harm to premature lungs. Animal studies have reported that inappropriately high pressure in the DR is associated with significant lung damage even when pressure was introduced for a few breaths. ${ }^{17}$ A recent trial on the use of sustained PEEP was discontinued due to lack of safety with potential harms with such approach. ${ }^{18}$

The interface used in this b-CPAP bundle was a specific type of binasal prongs that are wide, short and curved to accommodate the natural nasal passages (Hudson CRI, New Jersey or Babi.Plus, Carlsbad, CA). This type of prongs can fit snuggly into nasal apertures without imposing pressure on the nasal septum. Other types of prongs that are single, longer or narrower increase airway resistance and work of breathing. The use of nasal mask can be gentler to the nasal septum; however, the inherent leak around the septum is inevitable thereby providing inefficient pressure.

Infants were weaned off CPAP directly to room air and did not receive oxygen via nasal cannula. This is one of the major characteristics of the b-CPAP bundle. This practice guideline was initiated based on an animal study that showed increased lung growth with longer use of CPAP; the continuous distending pressure generated by CPAP was associated with increased lung volume, weight, cytoplasm and DNA contents in male ferrets after 2 weeks of therapy. This lung growth effect was not achievable after 1 week of therapy, which indicates that the stimulation of lung growth by CPAP requires time. ${ }^{19}$ In a subsequent clinical trial, weaning off CPAP prematurely with transitioning to nasal cannula was associated with a longer duration of oxygen use when compared to continuing the use of CPAP until oxygen is no longer required. ${ }^{20}$ A recent trial demonstrated the effect of extended use of CPAP on lung growth; premature infants that randomly received extra 2 weeks of CPAP had doubled their functional residual capacity when compared to controls. ${ }^{21}$ The use of nasal cannula early in the course of RDS is not helpful either. When compared to CPAP, the use of nasal cannula to support premature infants with RDS was determined to be an inferior therapy in a 
Table 2. Outcomes among VLBW infants by birth weight category.

\begin{tabular}{|c|c|c|c|c|c|}
\hline & $\begin{array}{l}\text { All infants } \\
<1500 \mathrm{~g} \\
n=773\end{array}$ & $\begin{array}{l}\text { Infants } \\
1000-1499 \mathrm{~g} \\
n=397\end{array}$ & $\begin{array}{l}\text { Infants } \\
750-999 \mathrm{~g} \\
n=162\end{array}$ & $\begin{array}{l}\text { Infants } \\
500-749 \mathrm{~g} \\
n=165\end{array}$ & $\begin{array}{l}\text { Infants } \\
<500 \mathrm{~g} \\
n=49\end{array}$ \\
\hline \multicolumn{6}{|l|}{ Primary outcomes } \\
\hline Mortality & 13.3 & 1.3 & 10.5 & 33.3 & 53.1 \\
\hline Transferred out & 15.4 & 9.9 & 15.4 & 26.1 & 24.5 \\
\hline Chronic lung disease (CLD) & 6.4 & 2.2 & 6.3 & 16.3 & 50.0 \\
\hline Survival to $36 \mathrm{wk}$ without CLD & 80.2 & 96.5 & 84.0 & 49.6 & 20.9 \\
\hline \multicolumn{6}{|l|}{ Secondary outcomes } \\
\hline Pneumothorax & 6.6 & 3.5 & 9.3 & 10.3 & 10.2 \\
\hline Pulmonary hemorrhage & 4.9 & 0.8 & 4.9 & 12.7 & 12.2 \\
\hline Necrotizing enterocolitis & 8.7 & 4.3 & 11.7 & 15.2 & 12.2 \\
\hline Intraventricular hemorrhage & 17.9 & 9 & 20.3 & 35.3 & 37.1 \\
\hline Severe IVH (>grade 2) & 6.2 & 2.1 & 5.1 & 15.4 & 20.0 \\
\hline Periventricular leukomalacia & 3.0 & 1.1 & 2.9 & 7.1 & 19.0 \\
\hline Hydrocephalus & 5.6 & 1.8 & 5.3 & 17.7 & 8.7 \\
\hline Retinopathy of prematurity & 16.8 & 6.2 & 25.2 & 37.4 & 52.4 \\
\hline Severe ROP (>stage 2$)^{\mathrm{a}}$ & 2.7 & 0.3 & 2.2 & 9.1 & 19.1 \\
\hline Hospital length of stay, day ${ }^{b, c}$ & $57 \pm 28$ & $44 \pm 19$ & $74 \pm 19$ & $93 \pm 24$ & $108 \pm 30$ \\
\hline Weight at discharge, $\mathrm{g}^{\mathrm{b}, \mathrm{c}}$ & $2413 \pm 519$ & $2321 \pm 475$ & $2556 \pm 549$ & $2581 \pm 570$ & $2744 \pm 521$ \\
\hline Head circumference at discharge, $\mathrm{cm}^{\mathrm{b}, \mathrm{c}}$ & $31.7 \pm 2.6$ & $31.4 \pm 2.3$ & $32.0 \pm 3.2$ & $32.4 \pm 2.5$ & $32.7 \pm 2.1$ \\
\hline Length at discharge, $\mathrm{cm}^{\mathrm{b}, \mathrm{c}}$ & $44.8 \pm 3.4$ & $44.6 \pm 3.1$ & $45.0 \pm 4$ & $45.1 \pm 4$ & $45.8 \pm 2.7$ \\
\hline
\end{tabular}

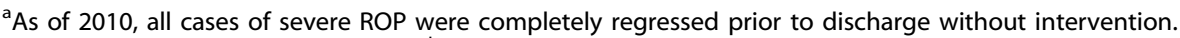

Data are presented in \% except with ${ }^{b}$ data are presented in median (IQR) and with ' data are presented in mean \pm s.d.

Table 3. Comparison of NICU outcomes with Vermont Oxford Network for infants (501-1500 g).

\begin{tabular}{lllll}
\hline & $\begin{array}{l}\text { GW NICU } \\
n=196\end{array}$ & Vermont Oxford Network & $p$ value & Risk difference-95\% (confidence limits) \\
\hline Mortality & 8.2 & 10.9 & 0.47 & $(-0.17)-0.11$ \\
Chronic lung disease (CLD) & 4.8 & 28.6 & $<0.001$ & $(-0.37)-(-0.09)$ \\
Necrotizing enterocolitis (NEC) & 4.1 & 5.2 & 0.73 & $(-0.15)-0.13$ \\
Severe intraventricular hemorrhage (IVH) & 6.5 & 7.9 & 0.78 & $(-0.15)-0.13$ \\
Severe retinopathy of prematurity (ROP) & 0.6 & 6.2 & 0.06 & $(-0.19)-0.09$ \\
\hline
\end{tabular}

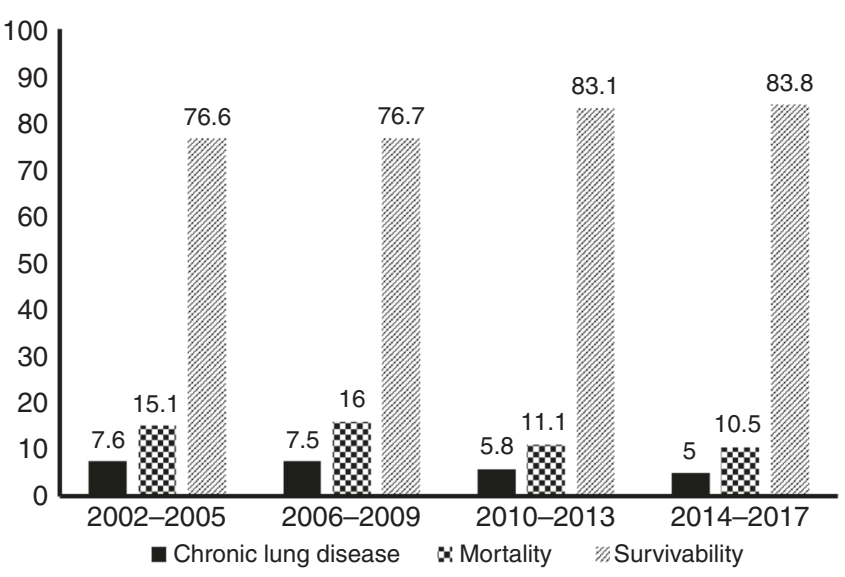

Fig. 1 Primary outcomes over 4-year intervals. recent multicenter randomized trial. ${ }^{22}$ One of the troubles associated with the use of nasal cannula is the lack of pressure control; studies measured a wide range of pressure $\left(0-14 \mathrm{cmH}_{2} \mathrm{O}\right)$ in the pharynx of infants supported with nasal cannula. ${ }^{20}$ Some nasal cannula devices allow adequate heating and humidification, although many of them will display significant water rain-out that leads practitioners to use colder and less humidified gas. Relatively dry and cold gas can add to the reactivity of respiratory airway especially those with existing lung disease. ${ }^{23}$ In addition, the bundle includes a careful attention to the patency of nasal and nasopharyngeal airway through frequent suctioning. Upper airway suctioning could be a challenging task especially in the smallest $\mathrm{GA}$ categories $\leq 24$ weeks; it has to be efficient to clear all secretions, gentle not to cause any trauma, and fast to avoid apnea and oxygen desaturation. Thus, bedside training and experience are crucial for the success of the bubble CPAP bundle. Based on the above, our cohort had some infants who received bCPAP without $\mathrm{O}_{2}$ supplementation at 36 weeks. Based on the definition of CLD used in major randomized controlled trials and 
Table 4. Respiratory management in the first 3 days of life by birth weight category (infants expired or transferred within first 3 days of life were excluded).

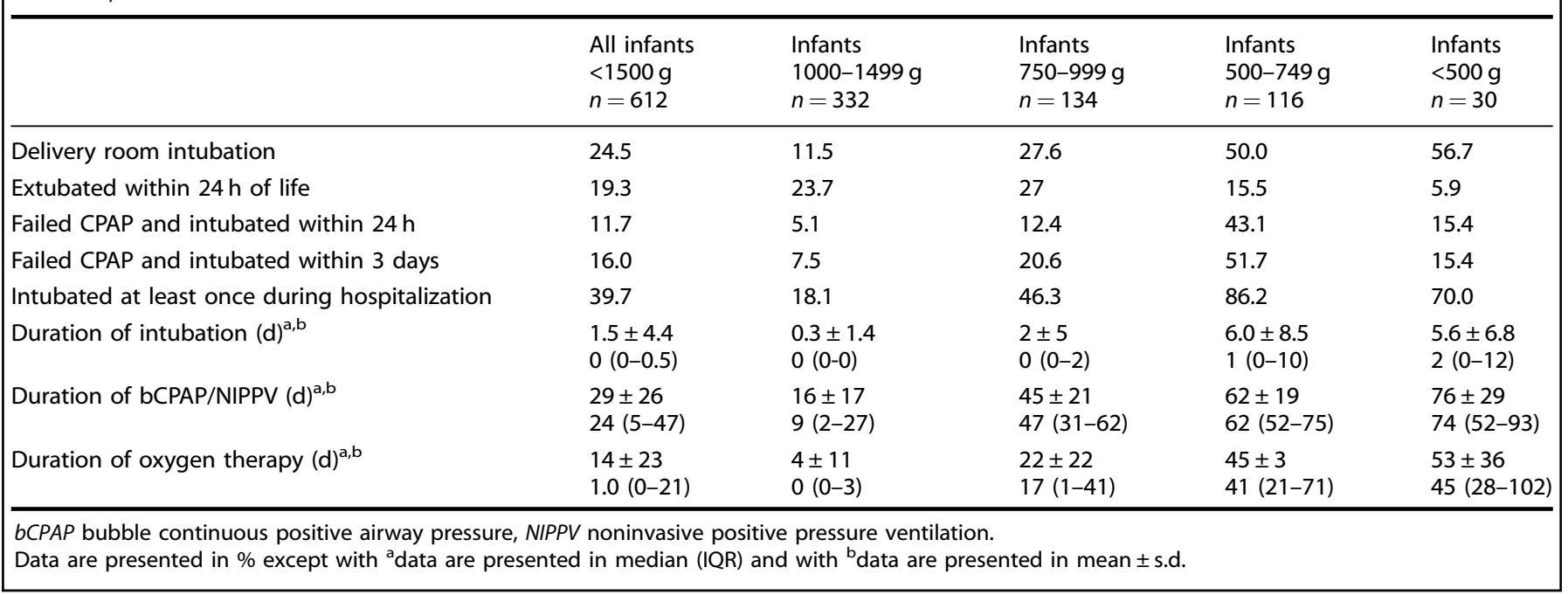

in benchmark reports, these infants were not classified to have CLD. Of note, the definition of CLD with $\mathrm{O}_{2}$ use at 36 weeks may not necessarily reflect long-term respiratory outcomes.

The prevalence of severe ROP was minimal in this cohort. None of the infants in the cohort received any laser or anti-VEGF therapy since 2010. The vast majority of infants received treatment for ROP prior to 2010 were those $\leq 24$ weeks of GA and typically received mechanical ventilation early in the NICU course (data not presented). It is plausible that the ventilator-associated inflammatory cascade may contribute, in addition to CLD, to retinal injury that leads to ROP. It has been recently recognized, in animal model, that mast cell activation is responsible for the abnormal neovascularization of the retina that leads to ROP; mouse strains lacking mast cells do not develop ROP when challenged with hyperoxia. ${ }^{24}$ Another explanation to the absence of ROP for several years could be the application of a tighter goal oxygen saturation range that possibly minimized episodes of hypoxemia.

The study carries multiple strengths. First, it demonstrates that a single-digit incidence of CLD is feasible and sustainable. Such finding is essential to be recognized to overcome the frustrations among caregivers when CLD incidences did not substantially decrease over the past few decades despite multiple randomized trials using various management strategies and therapies. ${ }^{4,5}$ Second, it introduces the important concept of "bubble CPAP bundle". Many of the studies conducted on the use of CPAP did not discriminate among various techniques of CPAP delivered, the type of nasal interface, and protocol of care at the bedside. Therefore, it is critical to include these details in future trials. Third, the study brings with it the challenge of whether this experience is reproducible and, if not reproducible, obstacles against its reproducibility would need to be addressed.

However, being retrospective, it is not possible to prove a cause-and-effect for the bundle to lead to the low rates of CLD. In addition, the study did not provide information whether the bubble CPAP bundle is the best approach for all GA and BW categories. For example, the prevalence of CLD in infants with GA $<24$ weeks was $46 \%$ and in infants with BW $<500 \mathrm{~g}$ was $50 \%$. There is a possibility that a better approach than initial b-CPAP exists for this, at the edge of viability, population. The study reported 16 years of experience, although the investigators noted possible changes in practice, other discrete modifications in management could have been overlooked during such long duration of therapy. The prevalence of CLD was shown to be consistently low when analyzed in four increments of 4 years each. Therefore, changes that were not counted for did not impact CLD.

\section{CONCLUSION}

This study shows that the use of a bubble CPAP bundle is associated with a lower rate of CLD that was relatively consistent over a period of 16 years. In addition to the early use of b-CPAP in the delivery room and early extubation to b-CPAP, the bundle included the continuous use of CPAP until oxygen is no longer needed. The bundle utilized specific types of CPAP, nasal prongs and airway care strategy. The b-CPAP bundle requires hands-on training for bedside nurses on detailed care of airway through a comprehensive competency checklist. It also raises the awareness of caregivers on the importance of prompt extubation for infants initially intubated in the DR. Such training creates a new culture of gentle respiratory approach. Once this culture develops among staff, it becomes impossible to conduct an ordinary RCT within that NICU. A cluster randomized trial, that randomly enrolls some NICUs in this training while allowing the rest of the NICUs to continue their conventional practice as a control, will prospectively test the efficacy of the b-CPAP bundle.

\section{AUTHOR CONTRIBUTIONS}

H.A. and M.A.M. conceptualized and designed the study, coordinated and supervised data collection, carried out the initial analyses, drafted the initial manuscript, and reviewed and revised the manuscript.

\section{ADDITIONAL INFORMATION}

The online version of this article (https://doi.org/10.1038/s41390-020-0763-3) contains supplementary material, which is available to authorized users.

Competing interests: The authors declare no competing interests.

Publisher's note Springer Nature remains neutral with regard to jurisdictional claims in published maps and institutional affiliations.

\section{REFERENCES}

1. Cheong, J. L. Y. et al. Changing neurodevelopment at 8 years in children born extremely preterm since the 1990s. Pediatrics 139, e20164086 (2017). 
2. Doyle, L. W. et al. Ventilation in extremely preterm infants and respiratory function at 8 years. N. Engl. J. Med. 377, 329-337 (2017).

3. Aly, H., Mohamed, M. A. \& Wung, J. T. Surfactant and continuous positive airway pressure for the prevention of chronic lung disease: history, reality, and new challenges. Semin. Fetal Neonat. Med. 22, 348-353 (2017).

4. Finer, N. N. et al. Early CPAP versus surfactant in extremely preterm infants. $N$. Engl. J. Med. 362, 1970e9 (2010).

5. Morley, C. J. et al. Nasal CPAP or intubation at birth for very preterm infants. $\mathrm{N}$. Engl. J. Med. 358, 700-708 (2008).

6. Perlman, J. M. et al. Part 11: neonatal resuscitation: 2010 international consensus on cardiopulmonary resuscitation and emergency cardiovascular care science with treatment recommendations. Circulation 122, S516-S538 (2010).

7. Schmolzer, G. M. et al. Non-invasive versus invasive respiratory support in preterm infants at birth: systematic review and meta-analysis. BMJ 347, f5980 (2013).

8. Van Marter, L. J. et al. Do clinical markers of barotrauma and oxygen toxicity explain interhospital variation in rates of chronic lung disease? Pediatrics 105, 1194-1201 (2000)

9. Aly, H., Massaro, A. N., Patel, K. \& El-Mohandes, A. A. E. Is it safer to intubate premature infants in the delivery room? Pediatrics 115, 1660-1665 (2005).

10. Carlo W. A. Gentle ventilation: the new evidence from SUPPORT, COIN, VON, CURPAP, Colombian Network, and Neocosur Network trials. Early Hum Dev. (Suppl 2), 88, S81-S83 (2012).

11. Aly, H. \& Mohamed, M. A. in Atlas of Procedures in Neonatology 5th edn (eds MacDonald, M. G. \& Ramasethu, J.) (Lippincott, Williams \& Wilkins, Philadelphia, 2012).

12. Mohamed, M. A. et al. Patent ductus arteriosus in premature infants: to treat or not to treat? J. Perinatol. 37, 652-657 (2017).

13. El-Dib, M., Narang, S., Lee, E., Massaro, A. N. \& Aly, H. Red blood cell transfusion, feeding and necrotizing enterocolitis in preterm infants. J. Perinatol. 31, 183-187 (2011).
14. Horbar, J. D., et al. Variation in performance of neonatal intensive care units in the US. JAMA Pediatr. 171, e164396 (2017).

15. Claassen, C. C., Hillman, N. H., Brown, K., Williams, H. L. \& Strand, M. L. Comparison of bubble CPAP devices using RAM cannula for extubation failure in very low birth weight infants: randomized and cohort studies. Neonatology 115, 28-35 (2019).

16. Courtney, S. E., Kahn, D. J., Singh, R. \& Habib, R. H. Bubble and ventilator-derived nasal continuous positive airway pressure in premature infants: work of breathing and gas exchange. J. Perinatol. 31, 44-50 (2011).

17. BjÖrklund, L. J. et al. Manual ventilation with a few large breaths at birth compromises the ther-apeutic effect of subsequent surfactant replacement in immature lambs. Pediatr. Res. 42, 348-355 (1997).

18. Foglia, E. E. et al. Sustained aeration of infant lungs (SAIL) trial: study protocol for a randomized controlled trial. Trials 16, 95 (2015)

19. Zhang, S., Garbutt, V. \& McBride, J. T. Strain-induced growth of the immature lung. J. Appl. Physiol. 81, 1471-1476 (1996).

20. Abdel-Hady, H., Shouman, B. \& Aly, H. Early weaning from CPAP to high flow nasal cannula in preterm infants is associated with prolonged oxygen requirement: a randomized controlled trial. Early Hum. Dev. 87, 205-208 (2011).

21. Lam, R. et al. The effect of extended continuous positive airway pressure on changes in lung volumes in stable premature infants: a randomized controlled trial. J. Pediatr. https://doi.org/10.1016/j.jpeds.2019.07.074 (2019).

22. Roberts, C. T. et al. Nasal high-flow therapy for primary respiratory support in preterm infants. N. Engl. J. Med. 375, 1142-1151 (2016).

23. Greenspan, J. S., DeGiulio, P. A. \& Bhutani, V. K. Airway reactivity as determined by a cold air challenge in infants with bronchopulmonary dysplasia. J. Pediatr. 114 452-454 (1989).

24. Matsuda, K. et al. Mast cell hyperactivity underpins the development of oxygeninduced retinopathy. J. Clin. Invest. 127, 3987-4000 (2017). 